\title{
Pour un dépassement des théories du game et du
} play

Going beyond Theories of Game and Play

\section{Brice Roy}

\section{OpenEdition}

\section{Journals}

Édition électronique

URL : https://journals.openedition.org/sdj/1709

DOI : $10.4000 /$ sdj. 1709

ISSN : 2269-2657

\section{Éditeur}

Laboratoire EXPERICE - Centre de Recherche Interuniversitaire Expérience Ressources Culturelles Education

\section{Référence électronique}

Brice Roy, "Pour un dépassement des théories du game et du play », Sciences du jeu [En ligne], 11 |

2019, mis en ligne le 16 avril 2019, consulté le 12 décembre 2022. URL : http://

journals.openedition.org/sdj/1709; DOI : https://doi.org/10.4000/sdj.1709

Ce document a été généré automatiquement le 12 décembre 2022.

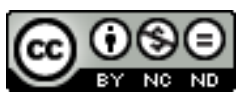

Creative Commons - Attribution - Pas d'Utilisation Commerciale - Pas de Modification 4.0 International - CC BY-NC-ND 4.0

https://creativecommons.org/licenses/by-nc-nd/4.0/ 


\title{
Pour un dépassement des théories du game et du play
}

Going beyond Theories of Game and Play

\author{
Brice Roy
}

1 Le jeu est une notion qui ne se laisse pas circonscrire. Le jeu est semblable au sable : il nous file entre les doigts. Le nombre de tentatives existantes pour le définir en est la meilleure illustration. Cette difficulté a pu être comprise comme la conséquence d'un piège tendu par le langage : ce qui poserait problème ne serait pas l'évanescence de la notion, mais la pluralité des phénomènes rapportés au mot, phénomènes qui ne partageraient en définitive qu'un «air de famille", pour reprendre la célèbre expression de Wittgenstein. Si nous nous attardons sur les principales définitions existantes, on peut néanmoins les organiser en deux groupes me semble-t-il. D'un côté, on trouve ces définitions dont le point commun est de comprendre le jeu comme une activité (Château, 1950), une expérience (Caillois, 1967) ou encore une attitude (Henriot, 1989). Dans cette approche, le jeu se voit avant tout compris comme une spécificité humaine, voire comme un indicateur de ce qu'a l'humain en propre. D'un autre côté, on distingue un certain nombre de théories où le jeu est approché comme un objet matériel, un ensemble de règles (Juul, 2005) ou encore un dispositif. L'objet de cet article n'est pas de prendre position en faveur de l'une ou l'autre de ces approches. Pas plus ne s'agit-il de proposer une énième solution pour les réconcilier. Il s'agit de s'intéresser aux raisons de leur antinomie et de se demander de quoi ces difficultés sont l'expression. Nous faisons cette proposition : par-delà leurs différences et oppositions, ces théories s'appuient sur une même préconception, l'idée d'une opposition de l'homme à la technique. Parce qu'elles s'inscrivent dans ce clivage sans en questionner les présupposés, les théories du jeu se résument le plus souvent à répondre à cette question : faut-il comprendre le jeu comme un objet technique ou au contraire comme un propre de l'humain? La controverse opposant les théories du game et du play depuis la deuxième partie du $\mathrm{XX}^{\mathrm{e}}$ siècle jusqu'à aujourd'hui en est l'illustration la plus manifeste. 
2 Ce qui fait défaut à la plupart des théories du jeu, c'est un socle solide pour approcher autrement la question de l'homme et de la technique. En guise de socle, nous proposons de nous appuyer sur la thèse dite « de la technique anthropologiquement constitutive et constituante » (ou thèse «TAC »). Plutôt que de penser l'homme et la technique dans un rapport d'opposition, cette théorie entend montrer en quoi la technique n'est pas seulement une production humaine, mais son corrélat. L'anthropologue Jack Goody, précurseur de cette école de pensée, montre ainsi que les supports d'écriture ne sont jamais de simples moyens d'expression, mais une condition de possibilité technique de la rationalité, explicitée dès lors en son caractère graphique (Goody, 1979). S'appuyant sur la découverte en paléoanthropologie d'une préséance de la technique sur l'humain (Leroi-Gourhan, 1943), le philosophe Bernard Stiegler (1994) propose plus radicalement de définir l'homme comme un animal prothétique marqué par un défaut d'essence. Un paradoxe y est assumé : que le propre de l'homme soit à chercher dans la relation qu'il noue avec la technique, rencontrée comme un support venant compenser et fonder un défaut de qualité originaire.

3 C'est en s'appuyant sur ce cadre théorique que nous nous proposons de revisiter la question du jeu. Pour ce faire, le jeu vidéo sera notre objet de prédilection. Celui-ci dispose en effet de multiples avantages pour le philosophe. C'est un objet nouveau, propice à l'étonnement. C'est un objet dont la dimension technologique peut difficilement être pondérée, tout en offrant des régimes d'expérience inédits (Triclot, 2011). On voudrait montrer en quoi la thèse «TAC » permet d'en tirer des perspectives philosophiquement fécondes et stimulantes. Soulignons que l'enjeu sera essentiellement programmatique: identifier quelques pistes, des lignes de faille, chacune susceptible d'encourager de plus amples développements.

Le présent article sera organisé de la manière suivante. Nous commencerons par montrer comment, chez Platon et Aristote, la figure du jeu se trouve mobilisée pour illustrer deux régimes d'activité qu'il s'agit d'opposer : la technè et l'epistemè. Nous montrerons ensuite comment cette approche normative du jeu se retrouve et se cristallise au $\mathrm{XX}^{\mathrm{e}}$ siècle, dans cet autre dualisme qu'est l'opposition de l'homme à la technique. Dans la dernière partie de l'article, nous montrerons en quoi la mobilisation de la thèse "TAC », en raison de la conception renouvelée qu'elle propose de l'homme et de la technique, offre des perspectives enthousiasmantes pour la fondation d'une nouvelle entente du jeu,et du jeu vidéo en particulier.

\section{Le clivage homme / technique}

5 Commençons par aborder la manière dont la technique est traditionnellement définie. Nous pourrons alors mieux comprendre pourquoi il est d'usage d'y opposer l'humain. Ces quelques rappels permettront d'aborder avec un autre regard les conceptions canoniques du jeu, en philosophie antique d'une part, en philosophie contemporaine d'autre part. Nous n'évoquerons pas les définitions du jeu que la philosophie moderne propose. Mais on pourra par exemple s'intéresser au caractère antithétique des définitions leibniziennes et kantiennes. On y retrouverait un partage analogue.

6 La technique désigne initialement l'ensemble des procédés répétables mobilisés dans la réalisation d'actions déterminées. Dans une telle acception, technique, pratique et manière de faire sont synonymes. C'est en ce sens que l'on peut parler de techniques de chasse ou de techniques d'écriture. Par extension, la technique désigne les artefacts 
que de tels procédés permettent de constituer, artefacts qui à leur tour peuvent être employés dans la réalisation de certaines actions. Outils, instruments et machines relèvent de cette catégorie. Leur mobilisation permet l'amplification de l'impact (principe de percussion), la prolongation du geste (principe de propulsion) et sa substitution (principe d'automatisation) (Leroi-Gourhan, 1943). Sous cette perspective, pratiques et objets techniques valent comme des supports, c'est-à-dire des éléments sur lesquels on peut prendre appui. Si la technique se présente alors comme un pouvoirfaire, ce pouvoir-faire est indissociable du savoir-faire qui rend possible sa mobilisation. C'est pourquoi la technique renvoie en outre aux règles, codes et procédures associés aux opérations techniques. A cet égard, la technique ne garantit pas seulement la conformité du résultat, mais aussi de la manière dont les choses sont conduites.

7 Parce que la technique offre la possibilité d'obtenir des résultats initialement considérés comme hors de portée, il est fréquent de la comprendre comme un outil. Ce faisant, on assimile la technique à sa disponibilité et aux résultats qu'elle permet d'obtenir. Imperceptiblement, c'est une conception utilitariste et productiviste de la technique qui peut s'installer. La technique se trouve assimilée à un moyen dénué de tendance propre, en attente des prescriptions venant lui assigner son orientation. Elle apparaît comme un moyen mis en œuvre par un agent extérieur, en direction d'un but qui ne lui appartient pas.

8 Cette vision de la technique est le pendant logique d'une caractérisation de l'humain située aux antipodes. Quand la technique est pensée comme un objet artificiel disponible pour l'usage, l'homme est compris comme un sujet libre par nature, capable de s'assigner à lui-même ses propres finalités. Si la relation de l'homme à la technique peut ensuite être questionnée, elle se réduit à examiner la manière dont cette dernière vient modifier un ensemble de possibilités considérées comme pré-données. En approchant les choses de la sorte, on fait de la technique un phénomène secondaire, en ordre et en importance. Elle apparaît en outre comme un ajout, un élément externe pouvant s'adosser à l'humain mais ne jouant aucun rôle dans la définition de son être. De cette conception résulte plus généralement un dualisme, autour duquel les couples esprit/matière, nature/artifice, intériorité/extériorité, liberté/déterminisme, sujet/ objet ou encore théorie/pratique viennent s'agencer.

9 La pensée grecque est un des lieux où se préfigure cette opposition. On fait ici référence à la séparation opérée chez les Grecs entre technè et epistemè, scission qui coïncide avec la naissance de la philosophie et passe par le rejet de la sophistique dénoncée comme instrumentalisation du logos (Stiegler, 1994, p. 15). Si l'epistemè est souvent traduite par le mot "science", elle peut aussi être comprise comme un synonyme du savoir ou encore de la vertu. La technè est quant à elle généralement traduite par le terme de "technique", mais elle semble aussi renvoyer à la notion d'habileté, à la pratique artistique ou encore à l'artisanat. Il convient cependant de ne pas forcer l'opposition. Si l'on peut parler de scission qui s'opère, c'est que les philosophes grecs s'interrogent justement sur la nature d'un ensemble de pratiques qu'il n'est pas aisé de distinguer. C'est pourquoi le sens de l'epistemè et de la technè varie non seulement d'un philosophe à l'autre, mais aussi au sein d'un même corpus. C'est avec Aristote que les notions d'epistemè et de technè commencent à se stabiliser et à entrer en opposition (Aristote, 2014). C'est dans le cadre de ces grands mouvements de pensée que la figure du jeu se trouve mobilisée. Ballottée d'un pôle à l'autre, le jeu est tantôt 
donné en exemple pour clarifier ce qu'est le propre de la technè, tantôt pour illustrer ce qu'on lui oppose, l'epistemè.

\section{Jeu et technè chez Platon}

10 Chez Platon, la figure du jeu est le plus souvent convoquée à des fins comparatives. Deux acceptions peuvent être identifiées: la première fait du jeu une construction artificielle offrant l'illusion d'une liberté, la deuxième un procédé à caractère manipulatoire. Loin d'être mis en opposition à la technè, le jeu lui est assimilé, au point de rendre difficile l'identification de critères distinctifs.

La première acception peut être repérée à l'occasion de comparaisons effectuées dans les dialogues entre situations de jeu et situations de la vie mondaine. Dans les Lois, le soldat, dont la vie repose entre les mains du stratège, est assimilé à un pion sur un plateau de jeu. De même, le citoyen, dont l'existence est déterminée par les lois de la Cité, est comparé à une marionnette dont on tirerait les ficelles - une comparaison également employée pour qualifier le statut de l'homme dans son rapport au cosmos (Platon, 1854, p. 206). Si le soldat peut être comparé à un pion, c'est que ce dernier peut être déplacé ou supprimé selon le bon vouloir du joueur. Si le citoyen est semblable à une marionnette, c'est que les mouvements de cette dernière sont déterminés par la traction de fils invisibles. Le joueur lui-même - dont le destin est soumis au résultat d'un jet de dés - n'échappe pas à cette caractérisation.

Dans ce premier type de comparaison, le jeu est loin d'être compris comme une activité libre. S'il en a l'apparence, il est en réalité marqué par le déterminisme. Mieux, ces situations se distinguent par leur caractère artificiel. Il s'agit de constructions, qui dissimulent leur véritable nature. Comparer ces situations à des jeux permet au philosophe de souligner cet aspect. Si cette comparaison est possible, c'est que le jeu est lui-même compris comme une construction artificielle et déterminée. La chose n'est pas surprenante : il faut se rappeler que pour Platon, le jeu est un procédé mimétique, à l'instar des arts picturaux et de la poésie. Son propre est d'être une image, c'est-à-dire la copie d'une chose sensible, ce qui en fait la copie d'une copie. Eloignée du Vrai par trois degrés, l'image ne fait que reprendre l'apparence de la chose qu'elle imite, sans hériter de son essence (Platon, 1966, pp. 359-362). En tant qu'image, le jeu est un objet trompeur, un simulacre. Derrière la liberté de façade qu'il présente, il n'est qu'un agencement habile d'éléments. Nous retrouvons l'idée d'une proximité entre jeu et simulation, la dimension simulatoire du jeu étant ici comprise comme un effet résultant de procédés artificiels dissimulés.

13 La seconde acception s'illustre à trois occasions : lorsqu'il s'agit de comparer le jeu à la philosophie, au sophisme et à l'écriture. Si la première comparaison aboutit à l'identification de critères de démarcation, les deux autres débouchent sur l'affirmation d'une proximité essentielle. Si la philosophie n'est pas un jeu, bien qu'elle puisse donner l'impression de n'être qu'un babillage, c'est qu'elle ne se réduit pas à la mobilisation des opérations langagières qu'elle implique (Platon, 1967, p. 225). Mais la nature abstraite des enjeux qui sont les siens tend à la faire passer pour telle. Il en va de même pour la dialectique: outil de prédilection du philosophe, celle-ci présente le risque d'être mobilisé en dissociation du contexte qui lui fournit son sens et de se réduire à n'être plus qu'un jeu logique (Platon, 1966, p. 292). En revanche, la sophistique est bien assimilée à un jeu. C'est qu'à la différence du philosophe, le 
sophiste n'aspire à l'obtention d'aucune vérité. En cela, il n'est qu'un joueur virtuose, un jongleur de mots (Platon, 1966, p. 216). Quant à l'écriture, elle n'est, prise en ellemême, rien d'autre qu'une manière d'apposer des inscriptions, sans offrir la garantie de renvoyer à des connaissances. En soi, elle n'est qu'une manière de semer des graines à tous vents, un spectacle réjouissant mais vain (Platon, 1997, pp.181-183). Sans la science pour en guider l'usage, elle n'est que pure prouesse technique et jouissance de cette prouesse. Comme on voit, l'explicitation du caractère technique d'une activité donnée passe chez Platon par son rapprochement avec la figure du jeu.

\section{Le jeu chez Aristote : une activité libre opposée à la technè}

14 A bien des égards, la conception aristotélicienne du jeu vaut comme une figure inversée de celle proposée par Platon. Pour Aristote, le jeu est un autotélisme, une activité ne visant à rien d'autre qu'à son propre accomplissement. Il ne renvoie à aucune extériorité et c'est pourquoi il doit être qualifié d'inutile et d'improductif. Activité désintéressée et indéterminée par nature, le jeu procure un plaisir valant comme une fin en soi. En tant que tel, il est compris comme l'illustration d'une capacité humaine à s'affranchir de la logique impérieuse des besoins, à agir non pas en vue d'un bénéfice attendu, mais par pur plaisir de le faire. Cette vision du jeu comme activité désintéressée en fait dès lors l'expression d'un rapport insigne à la liberté.

De manière remarquable, cette inscription du jeu dans le domaine des activités autotéliques conduit à l'affirmation de son caractère non-technique. C'est que pour Aristote, la technè doit être comprise comme une activité intéressée, mobilisée en réponse à la pression des besoins. Mieux, jeu et technè se trouvent mis en opposition de manière explicite. Dans l'Ethique à Nicomaque, c'est comme exemple d'activité contraire à la technè que le jeu se trouve mobilisé (Aristote, 2014, p. 226). Le jeu est l'illustration d'une liberté humaine fondamentale, c'est-à-dire d'une capacité à s'émanciper du déterminisme que représente et rappelle la technè. Opposé à la technè, le jeu se voit rapproché de l'epistemè. L'un et l'autre sont définis comme des loisirs.

Ce rapprochement n'aboutira jamais à leur assimilation. Pour Aristote, la pratique du jeu est indifférente à la question du Bien, sans autre visée que la recherche de son propre maintien et du plaisir qui en découle. La capacité du joueur à oublier de prendre soin de son corps est donnée comme illustration de cette réalité. C'est pourquoi le jeu n'est pas considéré comme une activité vertueuse et fait l'objet d'un déclassement. Consécutivement, il se voit conçu comme activité frivole et superficielle, en tant qu'il apparaît dépourvu de positivité.

Loin de pondérer l'opposition du jeu à la technè, ce divorce renforce leur antagonisme. Quand la technè est comprise comme instrument privilégié de l'activité laborieuse, le jeu est pensé comme interruption du cours besogneux des choses. Le jeu n'est plus défini comme activité mais comme relâchement. Il apparaît comme ce moment où les outils sont délaissés au profit d'un moment de détente. Sous cette perspective, jouer est le privilège de ceux qui ne sont pas encore en mesure de travailler, de ceux qui sont trop épuisés pour cela et de ceux qui, à l'abri du besoin, préfèrent perdre leur temps plutôt que de rechercher le Bien Suprême. Cette deuxième étape de la caractérisation du jeu est remarquable. Elle aboutit à constituer une définition du jeu en creux. Le jeu cesse d'être défini positivement, pour être compris comme l'envers de la technè. Cette 
manière de définir le jeu persiste aujourd'hui, la liberté qu'on lui prête étant comprise comme une forme de désœuvrement, de vacance (vide).

\section{La scission d'une notion : game versus play}

Qu'est-ce que le « détour » par Platon et Aristote permet d'établir ? Que la question du jeu est toujours déjà modelée sur un certain partage de l'humain et de la technique. Les théories du jeu des $\mathrm{XX}^{\mathrm{e}}$ et $\mathrm{XXI}^{\mathrm{e}}$ siècles réactivent ces lignes de clivage qu'on pouvait déjà décerner. La modernité substitue à l'opposition entre technè et epistemè l'opposition entre science et technique. Placée sous l'autorité de la science, la technique se trouve comprise comme un moyen permettant l'obtention des résultats prescrits par la Raison, instrument permettant à l'Homme de se faire maître et possesseur de la Nature (Descartes, 1966, p. 168). Mieux, elle est comprise comme la mise en œuvre concrète et appliquée des connaissances théoriques dégagées par le progrès scientifique. Si la situation diffère fondamentalement, c'est aussi pour cela que le jeu n'est désormais plus mobilisé comme un simple exemple, mais comme l'objet même de la réflexion. Deux approches se distinguent. La première, la ludologie, se nourrit des thèses du structuralisme (Levi-Strauss, 1985), de la théorie de l'information (Von Neumann, 1996) et de la cybernétique (Wiener, 2014). Principalement représentée dans le milieu anglo-saxon (Juul, 2005; Salen \& Zimmerman, 2003; Frasca, 2007), cette théorie définit le jeu comme un objet formel structuré par des règles - le joueur étant lui-même pensé comme un opérateur du système et son expérience comme le produit de ce dernier. A l'opposé de cette tendance, une certaine tradition d'inspiration psychologique, sociologique et phénoménologique (Fink, 1966 ; Caillois, 1967 ; Henriot, 1969), pense le jeu comme une expérience, une attitude ou encore un vécu. Pour ces théories, le jeu se distingue d'abord par son sens, sens dont il ne serait possible de saisir la spécificité qu'en s'intéressant au vécu intérieur du joueur. Loin de conférer une importance aux éléments techniques de jeu (matériel, règles, procédés), elles considèrent ces derniers comme de simples supports disponibles pour l'usage ou encore comme des éléments venant porter atteinte à la liberté du jeu (Fink, 1966; Caillois, 1967 ; Henriot, 1969).

Aussi antagonistes soient-elles, ces théories ont en commun de partager l'idée d'une nécessaire distinction entre la dimension objective et la dimension subjective du jeu, c'est-à-dire d'un côté cela avec quoi l'on joue et de l'autre cela qui est vécu lorsqu'on joue. Chez les uns comme chez les autres, la question du jeu, pour être formulée de manière adéquate, doit partir d'une distinction préalable entre ces deux pôles. La difficulté notoire à définir le jeu serait la conséquence de cet amalgame, amalgame que l'imprécision du langage contribuerait à entretenir. La chose serait particulièrement manifeste dans la langue française ou allemande, pour ceci qu'elles ne disposent que d'un terme pour désigner les dimensions plurielles que peut revêtir le jeu. C'est pourquoi ces théories, quoiqu'il en soit du pôle qu'elles choisissent ensuite de favoriser, accordent leur privilège à la langue anglaise, laquelle dispose de deux termes au sens distinct. D'un côté, le mot game permet de désigner le cadre au sein duquel l'activité ludique a lieu, ou encore cela avec quoi l'on joue. De l'autre, le mot play désigne cela qui dans un jeu est fait, ou encore cela qui est vécu (playful). La langue anglaise disposerait d'un avantage certain : elle permettrait d'éviter toute confusion entre deux champs bien distincts. On appellera dans les pages suivantes théories du game (ou ludologie) les 
théories consistant à comprendre le jeu comme un système de règles à l'opposé des théories du play, terme qu'on réservera pour désigner les théories où le jeu est compris comme une expérience, une attitude ou encore un vécu. Mais ces deux pôles ne désignent-ils pas simplement, en dernière instance, la technique et l'homme, considérés du point de vue du ludique?

\section{Jouer avec « n'importe quoi » et « presque rien »} structure ou un système, mais comme une activité humaine. Le jeu est compris comme le fait d'un joueur, dont il s'agit de questionner le sens, dans son contenu et dans sa tournure. A bien des égards, les travaux de Jacques Henriot peuvent être considérés comme l'aboutissement de cette tradition intellectuelle. Nous nous y attardons quelque peu, afin de montrer en quoi à nouveau, c'est l'idée d'une opposition de l'homme à la technique qui structure la réflexion.

21 Pour Henriot, le jeu est une attitude de second degré. Dans le jeu, le joueur serait à ce qu'il fait sans y être, c'est-à-dire de manière distanciée, comme dans un écart. L'activité ludique aurait bien une réalité. Mais elle serait ressentie par le joueur comme fictive, ce qui revient à dire qu'elle serait, dans le temps même de son effectuation, irréalisée dans son sens. Plus précisément, Henriot définit le jeu comme un faire comme si. Le jeu serait bien une activité, mais dans le faire de cette activité viendrait se loger un acte de métaphorisation, par lequel la chose effectuée ne serait prise ni pour elle-même, ni pour autre chose qu'elle-même, mais comme si elle était ceci ou cela (Henriot, 1989, p. 155). Le jeu relèverait en cela de la feinte, dans la mesure où le joueur serait à son activité, sans ignorer le caractère hypothétique d'un tel mode de rapport.

La priorité accordée à la figure du joueur amène Henriot à comprendre le jeu comme un acte de la volonté partant de l'intériorité du sujet pour venir apposer un sens, par le libre exercice de l'imagination, sur ce qui existerait "au dehors" de manière autonome. Une telle conception valide la thèse traditionnelle en métaphysique d'un découpage entre sujet et objet, intériorité et extériorité, homme et monde. En cela, elle contrevient aux préceptes de la phénoménologie husserlienne et sartrienne, dont elle se réclame pourtant. Il résulte d'une telle approche l'identification du jeu à un acte mental capable de se déployer en toutes circonstances, sans préoccupation pour les contraintes du réel. Plus encore, un tel acte mental est compris comme réflexif. La posture de second degré caractéristique du jeu serait l'illustration du caractère conscient d'une telle activité.

Si le propre du jeu est à situer au niveau du joueur, c'est toujours chez Henriot après avoir tenté de le définir depuis ses dimensions matérielles, formelles et pratiques. La localisation du sens du jeu dans l'intériorité du sujet est présentée comme la conséquence de cette inférence impossible. Pour Henriot, matériels, règles et pratiques sont compris comme des éléments dont il est possible de se servir pour jouer, mais dépourvus de caractère ludique intrinsèque (Henriot, 1989, p.100, 106 \& 131). Semblable lecture revient à affirmer un primat du sens sur la structure, primat d'autant plus marqué que pour Henriot, le jeu est une activité capable de se servir de n'importe quelle chose pour se déployer. En tant que tel, le jeu brillerait par son éminente autonomie. Loin d'être déterminé par la technique, le jeu serait l'une des activités qui le serait le moins. 

expliquer le privilège que ces théories semblent accorder aux jeux mobilisant les supports les plus pauvres? Henriot a beau exprimer un intérêt particulier pour les outils de jeu, tout se passe néanmoins comme si les jeux dotés des éléments les moins nombreux et les plus simples remportaient un plébiscite particulier. A l'inverse, les jeux mobilisant des supports techniques ou technologiques plus lourds font l'objet d'une large omission, que ce soit les jeux vidéo (chose sans doute compréhensible, les premiers jeux grand public n'apparaissant que dans les années 70), les flippers (dont l'âge d'or est à situer dans les années 60) ou les jeux mécaniques (le babyfoot par exemple). Comment interpréter cette préférence? Si le jeu est bien cette activité capable de se déployer en toute circonstance, pourquoi serait-il moins susceptible d'advenir dans un cadre techniquement "chargé » que dans son contraire ?Cette préférence pour les jeux « simples » est en cela suspecte et suggère qu'in fine, de telles théories considèrent (à leur propre insu ?) les supports (matériels ou formels) comme un frein au libre déploiement du jeu. Plus précisément, Henriot semble tiraillé entre deux conceptions opposées du jeu. D'un côté, on remarque chez cet auteur un intérêt remarquable pour la question technique, en particulier au travers des outils et instruments de jeu. De l'autre, il n'en reste pas moins attaché à l'idée d'une liberté du jeu.

\section{Du joueur opérateur au jeu sans joueur}

27 A l'inverse, dans les théories du game, les ludologues définissent le jeu comme un ensemble de règles, une série d'instructions destinées à fixer des limites et instruire sur les procédures à suivre (Juul, 2005, p. 37). Les règles définissent un périmètre, une 
limite spatiale et temporelle au sein de laquelle des principes particuliers sont en vigueur. On trouve ici l'idée d'une capacité du jeu à produire une démarcation, la délimitation opérée par les règles ayant pour effet de produire une rupture. C'est en ce sens que Salen \& Zimmerman (2003, p. 95), reprenant Huizinga, parlent de cercle magique afin de désigner ce qui dans un jeu fonctionne comme un espace traversé par des règles qui dérogent aux principes habituels. En cela, les règles du jeu produisent un effet d'artificialisation, à la fois au sens où ce qui se déroule déroge aux codes habituels, et au sens où ce qui s'y déroule est suspendu dans sa possibilité à l'autorité de règles conventionnelles, produites de toute pièce, c'est-à-dire artificielles.

Pour les ludologues, un jeu ne se définit pas seulement par ses règles, mais par le mode d'organisation de celles-ci. Ces dernières doivent être agencées de manière à pouvoir se répondre les unes aux autres et former un tout organisé. Mais il ne suffit pas de définir le jeu comme un ensemble de règles organisées pour saisir sa spécificité. Après tout, de nombreux objets techniques peuvent être définis de la sorte (par exemple un système de lois ou encore des recettes de cuisine). Le jeu a en outre pour propre de fonctionner comme une machine autotélique (Salen \& Zimmerman, 2003, p. 88-89). Le propre du jeu serait d'être un système bouclé sur lui-même, ce qui interdirait de le penser comme un outil. Ce divorce entre fonctionnement et utilité serait un critère déterminant pour décider du caractère ludique d'un objet donné.

Une telle définition a pour effet d'évacuer la figure du joueur ou du moins de minimiser son importance. Pour défendre cette position, on s'en réfère à la répétabilité des règles, lesquelles peuvent être mises en œuvre à plusieurs reprises et par différents joueurs sans changer de contenu. Il en résulte l'idée que le joueur est avant tout un opérateur, en charge de faire fonctionner le système, c'est-à-dire de mettre en œuvre les règles. Une telle conception implique de ne s'intéresser qu'aux opérations de manipulation réalisées par le joueur (prendre une carte, déplacer un pion, passer son tour, etc). Le joueur se trouve réifié, assimilé lui-même au statut de variable. La possibilité de déléguer de telles procédures à une machine automatisée semble étayer la validité de ce point de vue. Or, il apparaît envisageable de déléguer à une machine la prise en charge de l'intégralité des actions de jeu, dans la mesure où celle-ci peut également être programmée pour appliquer un protocole de décision lorsque la règle de jeu autorise l'exécution d'un choix. Parce que le système de règles se "désintéresse » du statut de l'opérateur, il semble possible de définir ce qu'est un jeu sans faire intervenir le concept de joueur.

Cette position est adoptée par Juul dans le célèbre article "Zero-Player Games ». En conclusion, Björk et Juul défendent l'idée selon laquelle les jeux n'ont pas besoin d'être pratiqués par des personnes ; qu'un joueur n'est pas nécessairement un acteur humain, mais peut fort bien être une variable du système lui-même (Björk \& Juul, 2012). L'article aboutit à faire du jeu un objet technique " pur ", fonctionnel mais inutile et autonome vis-à-vis de l'humain. Le partage homme / technique est une fois de plus non seulement préservé, mais renforcé. On notera avec intérêt que pour arriver à cette conclusion, Björk et Juul prennent appui sur des systèmes automatisés, sans s'interroger sur la conception de la technique qu'ils valorisent : une construction artificielle, réduite à son pur fonctionnement, et comprise comme sans rapport nécessaire à l'humain.

31 On peut enfin s'intéresser aux conséquences d'une telle définition. En faisant de la règle et du fonctionnel le socle de sa conception du jeu, la ludologie se condamne à perdre toute prise sur ce qu'on appelle usuellement les jeux «libres». L'hémiplégie dont 
souffre la ludologie produit les résultats inverses de ce que nous observions avec les théories du play: non pas la disqualification des pratiques ludiques ayant explicitement affaire à la technique, mais des pratiques qui semblent les moins déterminées et les moins artificielles. Ainsi, les jeux des jeunes enfants brillent par leur absence dans les livres de ludologie.

\section{Le jeu vidéo, une opportunité de remise en question manquée} technique, les théories du jeu sont condamnées à opter entre les deux termes d'une alternative aussi inconciliables que mutuellement indispensables. Renoncer à qualifier l'importance de la technicité du jeu, c'est en faire une abstraction incapable de rendre compte du caractère concret des pratiques ludiques. Renoncer à penser le jeu dans son rapport à l'humain, s'est perdre de vue tout critère permettant de saisir sa spécificité. $\mathrm{Au}$ mieux, ces théories sont condamnées à renouer avec le piège de l'hylémorphisme : penser le pôle play comme une matière informée par la dimension structurante du game ou inversement, considérer le game comme ce matériau dont on pourra s'emparer pour lui assigner, par l'exercice de l'imagination, la forme souhaitée.

L'arrivée du jeu vidéo offre une opportunité remarquable pour qui chercherait à dépasser ces modèles. A la différence de jeux plus classiques, il apparaît encore à beaucoup comme une incongruité, y compris pour ses plus fervents adeptes. Il faut dire que l'objet est encore loin d'être stabilisé. En constante évolution, le jeu vidéo est une singularité, à la fois sur le plan technique et sur le plan expérientiel. D'un côté, les dispositifs vidéoludiques prennent appui sur des paradigmes techniques inédits. C'est en particulier le cas du numérique, que le jeu vidéo s'est approprié avec une aisance remarquable - au point d'inviter à se demander s'il ne s'agirait pas d'un matériau ludogène, c'est-à-dire propice à l'adoption d'une posture ludique (Vial, 2013).

De l'autre, les expériences qu'on y vit ne semblent pas relever des registres habituels. On pense aux observations de Mathieu Triclot concernant les catégories d'expérience ludiques de Caillois: dans un jeu vidéo, de nouvelles combinatoires émergent, permettant par exemple d'associer expérience du vertige (Ilinx) et de l'imitation (Mimicry) (Triclot, 2011, p. 120). Pour toutes ces raisons, le jeu vidéo est une source d'étonnement, ce qui représente un véritable avantage sur le plan philosophique. A la différence de jeux plus classiques, il est en mesure de nous déstabiliser, y compris dans nos habitudes de pensée les mieux ancrées. Loin de participer au dépassement des théories précédemment évoquées, le jeu vidéo semble pourtant les avoir renforcées.

Du côté des théories du play, le jeu vidéo tend plutôt à être passé sous silence. Comme évoqué précédemment, peut-être cette pratique n'était-elle alors pas assez visible pour susciter l'attention des chercheurs. Ou peut-être y avait-il difficulté, pour une certaine génération de chercheurs, à s'y pencher. Mais on peut aussi se demander si, au fond, les jeux vidéo n'étaient déjà pas soupçonnés de se substituer au travail d'imagination du joueur. Il faut par exemple se souvenir que pour Henriot, jouets et objets ludiques relèvent d'un imaginaire exogène (Henriot, 1989, p. 156) quand ce qui fait jeu pour le chercheur se situe dans l'intériorité du sujet et doit en ce sens être qualifié d'imaginaire subjectif : 
[...Le] projet qu'il convient d'élaborer est celui d'une recherche qui cesserait de vouloir obstinément détecter la présence du jeu dans le monde des choses telles qu'on les perçoit, mais s'attacherait à le découvrir dans le monde des choses telles qu'on les imagine (Henriot, 1989, p. 157). du cinéma (Adorno \& Horkheimer, 1944). Pour ces derniers, le cinéma, participerait d'une mise à mort de l'imagination, en ce qu'il fournirait des images artificiellement constituées venant se substituer à celles qu'aurait pu produire l'imagination du spectateur. La méfiance d'Henriot n'est-elle pas de même ordre ? En cela, le jeu vidéo serait l'aboutissement d'une histoire de la délégation par où l'humain en viendrait progressivement à se déposséder de son pouvoir d'imagination, au profit des supports technologiques. Loin d'être une source d'enrichissement, le jeu vidéo participerait ainsi d'un appauvrissement et d'une normalisation des représentations. Le jeu vidéo est identifié comme un objet ludique remarquable. Chez les ludologues, cela est si vrai que le jeu vidéo est pris comme objet-témoin (Salen \& Zimmerman, 2003 ; Frasca, 2007). Son examen permet d'identifier les caractéristiques qui font le propre du jeu. Pour le comprendre, il faut se rappeler combien la ludologie s'inspire de la théorie de l'information, de la cybernétique et du structuralisme (lui-même inspiré des travaux de Von Neumann), c'est-à-dire de disciplines ayant pris l'informatique pour objet de prédilection. Même si l'objet de la ludologie est d'établir un modèle théorique propre au jeu, le privilège du jeu vidéo est de se déployer en faisant appel à un matériau qui se définit lui aussi comme système de règles: le numérique. Ajoutons que le principe d'un jeu sans joueur, formulé par Björk et Juul (2012), doit beaucoup à l'étude des jeux vidéo : de manière encore plus marquée que les jeux électroniques et les jeux mécanisés, les jeux vidéo comportent en effet une grande part d'automatisation. Au lieu de se demander ce qui en résulte sur le plan de l'expérience, pour le joueur, ces théories y voient plutôt l'indice de sa disparition possible. C'est en ce sens que le joueur peut être compris comme un opérateur en charge de faire fonctionner le système, une approche qui n'est pas loin de faire du joueur un rouage (nouveau glissement) dont on peut alors envisager la supplétion.

Notre thèse est la suivante : tant que la question du jeu sera abordée à l'aide d'un modèle théorique réduisant la technique à son fonctionnement et à son utilité, on ne pourra parvenir à saisir ce qui fait la singularité du jeu. Or, la remise en question de ce modèle est loin d'être hors de portée. Nous faisons référence aux préceptes de la thèse de la technique anthropologiquement constitutive et constituante. Inaugurée par Jack Goody et Leroi-Gourhan, prolongée par Bruno Bachimont ou encore Bernard Stiegler, cette théorie se propose de caractériser la technique comme ce quoi sans lequel l'homme ne serait pas un qui. En d'autres termes, elle fait de la technique le support sans lequel l'homme ne serait pas ce qu'il est, un être en perpétuel écart par rapport à lui-même. Naturellement, l'exploitation à plein de ces théories en vue de proposer une conception renouvelée du jeu dépasse le cadre et l'économie de cet article. C'est pourquoi nous privilégierons l'identification de lignes de faille. Comme mentionné plus haut, le jeu vidéo sera notre objet de prédilection. Ce dernier présente plusieurs avantages. Premièrement, nous y sommes moins accoutumés : nous avons là un jeu qui fait contraste dans le paysage ambiant, suscitant encore aujourd'hui la polémique et la controverse. Le jeu vidéo inquiète et cette manière d'inquiéter a quelque chose d'étonnant, philosophiquement parlant. Deuxièmement, le jeu vidéo est un objet dont il 
est difficile de nier la double singularité : technologique et expérientielle. Les jeux vidéo font partie des dispositifs qui mobilisent avec le plus d'aisance ces technologies disruptives que sont l'électronique et le numérique. Mais ils ont aussi pour particularité remarquable de donner à vivre des formes d'expérience inédites. Mieux, ces deux volets apparaissent comme difficilement dissociables. On a donc là un objet dont on peut espérer qu'il nous facilite le travail.

\section{Non-neutralité des supports de jeu}

Une première manière de dépasser les cadres théoriques usuels consiste à faire appel aux thèses de Jack Goody sur la non-neutralité des supports techniques. Pour Goody, la rationalité n'est pas une faculté qui préexiste aux supports d'écriture mobilisés pour s'exprimer. Les opérations techniques propres à l'écriture (liste, tableau, formule, etc.) doivent être comprises comme les conditions de possibilité de la rationalité, dont elles favorisent l'émergence (Goody, 1989, p. 122, 140, 198). A titre d'exemple, citons le fait d'apposer des traces sur une page : une telle opération, neutre a priori, rend d'emblée possible la délinéarisation du discours et contraint l'esprit à s'interroger sur la manière de pouvoir exprimer, sur une surface restreinte, la même quantité d'information. De même, l'écriture favorise-t-elle l'émergence de la pensée systématique et la montée en abstraction (formalisme). Les supports d'écriture ne sont pas simplement des éléments venant modifier notre manière de réfléchir: ils sont à comprendre comme les conditions de possibilité d'une rationalité dès lors explicitée en son caractère toujoursdéjà graphique.

En continuité avec ces observations, on peut se demander ce que l'écriture nous fait, quand nous la mobilisons dans le cadre de pratiques ludiques. La simple évocation de ces questions ouvre de nombreuses pistes. Intéressons-nous à l'une d'entre elles: le statut du livret de règles. Spontanément, le livret se comprend comme un support permettant de consigner la manière dont il faut jouer, c'est-à-dire la mémoire de ce qu'il faut faire et ne pas faire. Mais il est alors nécessaire de noter une première conséquence: à partir du moment où l'on dispose d'une mémoire externe, la possibilité d'augmenter le nombre de règles se présente. La mobilisation de l'écriture favorise une certaine complexification des jeux. L'apparition des règles écrites pose en outre deux problèmes inédits : il est désormais nécessaire de s'orienter dans les règles (c'est-à-dire de prendre le temps de les parcourir) et de les interpréter. Cette double nécessité favorise deux tendances venant résorber ces difficultés: une montée en systématicité et en formalisme (l'avantage du formalisme étant la réduction de l'effort interprétatif). Une règle écrite ne doit pas seulement être comprise, elle doit être appliquée. Là aussi, cette nécessité a des conséquences dramatiques: plus un jeu contient de règles, plus le temps nécessaire à leur application devient conséquent, ce qui menace à tout moment d'entraîner un déséquilibre. Qui a déjà joué au jeu de société Dune (Avalon Hill, 1979), connu pour ses nombreuses règles et ses nombreuses exceptions, sait ce qui en résulte : le risque de passer l'essentiel de son temps non à jouer, mais à vérifier comment jouer.

41 Pour pallier le problème de la quantité de règles à connaître et à appliquer, une solution consiste à en déléguer la charge à une personne tierce. C'est la solution adoptée dans le jeu de rôle. Les règles du jeu y sont prises en charge par ce qu'on appelle le maître de jeu. Celui-ci connaît, interprète et applique les règles, libérant les 
joueurs de ces obligations. Dans la mesure où il n'est plus nécessaire que les joueurs connaissent l'intégralité des règles, il en résulte l'augmentation tendancielle de leur quantité et complexité. Cette tendance fait rapidement du maître de jeu un expert, une sorte de maître de savoir. Imperceptiblement, le livret de règles cesse d'être consulté par les joueurs : parce qu'ils n'en ont pas besoin et parce qu'ils ne peuvent s'y orienter. Le livret de règles devient-il un objet privé, en partie ou en totalité. On se retrouve à jouer à un jeu dont non seulement on ne connaît pas les règles (un comble pour un joueur de jeux de société), mais qu'il est interdit de consulter. Le principe de publicité des règles, allant-de-soi de tout jeu de société, est brisé.

Il faut ici penser aux travaux de Bruno Bachimont sur le statut du numérique. Parce que le numérique repose sur un principe de discrétisation et de manipulation, les documents numériques différent fondamentalement de documents réalisés sur des supports analogiques (Bachimont, 2010, pp.155-156). Les données d'un document numérique ne sont en elles-mêmes que des unités vides de sens (des 0 et des 1 ), dont il n'est possible de tirer un sens que par l'intermédiaire d'un protocole d'interprétation (lui-même numérique). Cette caractéristique du numérique a des conséquences importantes : elle implique l'impossibilité, pour un document numérique, de disposer d'une quelconque authenticité. L'exemple de l'appareil-photo numérique permet de le comprendre : à la différence d'un appareil argentique où la photo est le témoin de ce moment où la lumière a percuté la pellicule, un appareil numérique ne fait que rapporter un signal à une suite conventionnelle d'états binaires. La photo qui en résulte n'est plus le témoin d'un évènement passé. Son noème n'est donc pas le "ça a été " barthien, mais le "ça a été manipulé». (Bachimont, 2010, p. 158). Ces observations impliquent de se demander le sens que peut encore avoir la rationalité, quand celle-ci s'appuie désormais non plus sur la fiabilité de l'écriture, mais sur l'incertitude du numérique. Peut-on véritablement parler d'une raison computationnelle? Ou faut-il acter de la tendance du numérique à se déployer comme un « labyrinthe » de données dans lesquelles on se perd?

Dans la plupart des applications, cet aspect problématique du numérique est refoulé. Une encyclopédie en ligne peut se présenter sous les atours d'un document préservé de toute variation. Nous simulons, à l'aide du numérique, des contenus audiovisuels noninteractifs, occultant la manipulabilité du numérique. Nous écoutons des morceaux de musique conçus pour coller au mieux avec la qualité de l'analogique. Mais il n'en est pas de même pour le jeu vidéo. Le numérique offre un avantage remarquable pour le jeu : il permet la délégation non pas de la mémorisation des données (ce que permet l'écriture), mais de la manipulation des données, dont résulte concrètement l'exécution des règles. Cette possibilité a un prix : la discrétisation des données à manipuler. La conséquence est considérable. Elle implique de ne jamais pouvoir accéder, en tant que joueur, aux règles en tant que telles. Au mieux pourra-t-on avoir accès, à l'aide d'une interface (par exemple un écran), à une certaine présentation des données. Le numérique introduit un intermédiaire entre le support d'inscription et le support d'accès : le support d'interprétation (Bachimont, 2001, p. 12). Dès lors, ce ne sont jamais les données que l'on consulte, mais une certaine traduction de celles-ci. Cela vaut pour le jeu vidéo comme pour n'importe quel programme. De ce principe résulte une deuxième conséquence : la possibilité que les données du jeu auquel on joue évoluent. Entre deux parties de Super Mario Bros. (Nintendo, 1985), ou même au cours d'une seule et même partie, il m'est impossible de pouvoir affirmer avec certitude que les règles du jeu auquel je joue n'ont pas changé. Mieux encore, ce principe est au cœur de la 
jouabilité de nombreux jeux vidéo : soit le principe de jouer à un jeu dont on ne connaît pas les règles, soit celui de règles susceptibles d'évoluer en permanence. Ainsi, il devient possible de comprendre les jeux vidéo comme des dispositifs de jeu reposant sur la méconnaissance de cela à quoi on joue.

\section{De l'usine à la salle d'arcade}

Une deuxième manière de remettre en question la pertinence du clivage homme / technique consiste à s'intéresser aux enseignements prodigués par la technologie moderne.

La technologie se distingue par sa propension à produire des résultats indésirables. Les exemples ne manquent pas pour illustrer cet état de fait. On peut penser au système de transport automobile, lequel génère pollution, embouteillages et accidents; ou encore aux technologies de la communication, censées favoriser le développement de la vie sociale, mais qui peuvent tout aussi bien engendrer l'isolement. Cette réalité interdit de penser la technique comme nécessairement adéquate aux finalités qu'on peut lui assigner (Ellul, 2012).

La technologie a pour autre particularité de pouvoir nous échapper. L'augmentation indéfinie de la puissance technique, couplée à l'accroissement de son automatisation, favorise cette tendance. La technique n'apparaît alors plus comme quelque chose dont on se sert, mais comme quelque chose que l'on cherche à maîtriser. La nécessité d'une mainmise sur la technologie s'impose à partir du moment où les effets qui en résultent peuvent représenter une menace et nous entraîner vers la catastrophe. Là encore, les exemples ne manquent pas. On peut songer au nucléaire et ses dangers, ou encore aux robots militaires autonomes - susceptibles d'engager des conflits non choisis, y compris contre leur propre camp (Jonas, 1995).

a quelque chose de profondément ludique, comme cela est mis en lumière dans le film Playtime (Jacques Tati, 1967) et surtout dans Les Temps modernes de Charlie Chaplin (1936). On fait en particulier référence à la célèbre séquence de la chaîne de montage. On y trouve Chaplin affairé au montage de pièces détachées, lesquelles défilent sur un tapis roulant. Le comique et l'absurde de la scène tiennent à la cadence infernale à laquelle Chaplin est soumis, en raison de la vitesse de défilement du tapis. Le point ici important à noter, c'est que la vitesse du tapis ne va pas véritablement trop vite, sans quoi le personnage de Chaplin ne pourrait suivre. Elle va trop vite pour qu'il soit possible d'en suivre la cadence sans se contorsionner. Pour suivre la cadence, Chaplin doit rompre avec les habitudes et adopter une posture improbable. En d'autres termes, il doit inventer. Il y a là quelque chose qui semble en dire long sur la nature du ludique. Cela n'est pas sans faire écho à ce que sont par exemple la poésie ou le bricolage, des arts de faire avec les limites des ressources dont on dispose.

Aussitôt évoquée l'hypothèse d'une connexion entre objet technique dysfonctionnel et jeu, une autre manière de regarder les jeux vidéo s'impose. Prenons le jeu Dance Dance Revolution (Konami, 1998). Dans ce jeu vidéo, le joueur dispose d'un tapis de danse électronique divisé en cases, chaque case valant comme un bouton permettant d'interagir avec le système. Le but du jeu est d'appuyer en cadence sur les boutons, à mesure que les icônes correspondantes défilent sur l'écran. La situation est comparable à la séquence des Temps Modernes : le joueur se retrouve à sauter sur les boutons comme s'il travaillant à la chaine, luttant contre la tendance du système à accélérer. Mais pour 
saisir l'intérêt ludique de ce type de proposition, l'erreur n'est-elle pas de s'intéresser uniquement aux instructions qui défilent et à la nécessité de faire la bonne chose au bon moment? Si nous admettons que le jeu a affaire avec l'inventivité, on voit difficilement comment une telle situation a quoique ce soit d'inventif. A moins que nous n'ayons pas regardé au bon endroit : si en effet la partition qui défile ne suscite en elle-même nulle créativité, ce n'est en revanche pas le cas du dispositif tout entier. Regardons les joueurs jouer pour s'en convaincre : ces derniers multiplient les postures les plus improbables et, parvenus à un certain niveau de maîtrise, réalisent les chorégraphies les plus étonnantes. Est-ce alors du côté du tapis de danse qu'il fallait chercher? Pas plus. Ce qui apparaît, c'est que le caractère ludique du dispositif tient à sa dimension dysfonctionnelle ou plutôt sous-optimale : il s'agit là d'un dispositif qui n'est manifestement pas fait pour pouvoir appuyer sur des boutons au bon moment. Pourquoi ? Parce qu'il implique de se contorsionner. Plutôt que de penser les dispositifs de jeu comme des supports dont on se sert, peut-être est-il plus pertinent d'examiner en quoi la technique, en raison des tendances qui lui sont propres, pose des problèmes susceptibles d'intéresser un joueur. Les dispositifs ludiques seraient des dispositifs manquant de praticité et suscitant comme tel l'inventivité. Une possibilité qui rappelle la proposition de Bernard Suits assimilant le jeu à une suite de problèmes nonnécessaires $(2005$, p. 34). Si la technique est une chose susceptible de poser problème ou de nous déstabiliser, peut-être tire-t-elle sa capacité à faire jeu non de sa disponibilité, ou de son indisponibilité, mais de son inadéquation.

\section{Du mode d'existence des objets ludiques}

Une troisième méthode consiste à caractériser la technique non pas comme un objet, mais comme un processus. Telle est l'approche de Simondon (1958). Plutôt que de comprendre la technique à partir de son usage, il est possible de s'intéresser à son évolution dans le temps. Sous cette perspective, un artefact vaut d'abord comme le témoin localisé d'un processus évolutif souterrain. En s'intéressant à la place qu'occupe l'artefact au sein d'une lignée, il est possible d'inférer l'existence de tendances. Mais la technique n'est pas un processus vivant. Bien qu'il y tende, l'objet technique n'est jamais achevé, à la différence du vivant. En outre, il n'est rien sans l'humain pour le constituer. Un enjeu est alors d'identifier la manière dont l'objet technique évolue dans le temps et selon quels principes.

Là encore, on dispose d'une approche féconde pour penser le jeu à nouveaux frais. On songe par exemple au travail de Nicolas Nova, à l'endroit de la manette de jeu vidéo. Comme le montre Nova, la manette de jeu vidéo évolue en interaction avec son milieu. Initialement attachée à (et indissociable de) la borne d'arcade, elle s'en émancipe rapidement, favorisée en cela par son arrivée dans l'espace du salon. Seul le raccord filaire témoigne de son attachement d'origine. La manette évolue de manière à se prêter à la manipulation. Elle diminue en épaisseur, ce qui favorise la prise et induit en retour une modification de la position des doigts et des pouces. Cette nouvelle configuration ouvre la possibilité de voir se multiplier les boutons sur le haut du boitier. A partir des années 90, une tendance de la manette est de prendre une forme en "boomerang ", ce qui vient libérer les index, permettant l'adjonction de boutons sur les côtés (Nova, 2013). 
51 Un autre bénéfice de l'approche simondonienne est de nous rappeler l'absence de connexion nécessaire entre objet technique et utilité. Si rétrospectivement, nous avons l'impression qu'un objet technique évolue de manière à répondre à un besoin, nous savons qu'il n'en est rien. Bien souvent, les inventions précèdent les usages qui leur correspondent, pour cela que l'invention technique fait émerger des besoins qui ne lui préexistaient pas. Or, là où cet aspect nous intéresse, c'est qu'il permet de dresser des ponts entre inventions techniques et objets ludiques. Si nous y prêtons attention, nous pouvons en effet remarquer ceci : qu'une technologie nouvellement constituée, bien loin de se présenter comme utile, se trouve employé comme un jouet. La chose est manifeste chez les enfants, qui de s'amuser avec le téléphone, qui de s'amuser avec le phonographe. Ce qui amuse à chaque fois, c'est la distorsion amenée par l'objet technique, le décalage qu'il produit au regard d'un environnement jusque-là banalisé. Ce phénomène est tout autant à l'œuvre chez les adultes à l'arrivée d'un nouvel objet technique dont on ne sait quoi faire, mais qui nous ravit par les possibilités qu'il semble promettre. Il nous déstabilise, nous désoriente, participe d'une reconfiguration de notre rapport au monde. Au fond, un objet ludique ne serait-il pas un objet technique ayant pour effet d'empêcher ou retarder le retour du banal, c'est-à-dire de nous acclimater? Sous cette perspective, la question n'est plus de savoir quelle est la particularité des objets techniques avec lesquels on joue, mais de comprendre quelles sont les conditions à partir desquelles un objet ludique cesse de nous amuser.

\section{Jeu et désorientation}

Enfin, il est possible de s'appuyer sur le travail de Bernard Stiegler. La postphénoménologie stieglérienne s'inscrit dans le prolongement des travaux du paléoanthropologue Leroi-Gourhan et de l'ontologie heideggérienne, qu'elle cherche à articuler. Rappelons tout d'abord la découverte de Leroi-Gourhan : soit la préséance de la technique sur l'homme, la technique accompagnant et évoluant de concert avec le processus d'hominisation menant du Zinganthrope à Homo Sapiens (Leroi-Gourhan, 1943). Pour Stiegler, ce qui se joue dans ce processus est l'avènement d'un nouveau type d'évolution: l'épiphylogenèse (Stiegler, 1994, p. 185). Ni génétique, ni épigénétique, l'épiphylogenèse désigne le principe d'une évolution par la transmission des caractères culturels, transmission que permettent les supports techniques, mais cela au prix de leur mise à mort (mémoire morte). Cette nouvelle forme de transmission implique de l'humain qu'il soit toujours-déjà sorti de lui-même, en inadéquation vis-à-vis des externalités techniques qu'il constitue, lui survivent et qui comme telles le précèdent.

En quoi cette conception est-elle susceptible de donner lieu à une conception du jeu renouvelée ? C'est que parmi les différents objets techniques existant, les dispositifs de jeu forment me semblent-ils une exception. A la différence des objets culturels en particulier, les supports de jeu ont pour particularité de ne pas engrammer la mémoire des pratiques passées, ou plus précisément de provoquer leur effacement. C'est en tout cas une chose remarquable pour certains dispositifs ludiques, comme le bac-à-sable par exemple. A l'instar de bien d'autres supports, le bac-à-sable peut être vu comme un support d'écriture. Mieux encore, le matériau mis à disposition se présente comme hautement disponible pour la manipulation et se prête à toutes les configurations. C'est pourquoi les bac-à-sable sont considérés parmi les supports de jeu les plus propices à l'exercice de l'imagination. Mais le sable dispose comme on sait d'une autre particularité : les réalisations qu'on peut constituer ont pour particularité de ne pas 
tenir. Elles se délitent. En cela, le sable est marqué par un manque de rétentionalité. Mais si nous poursuivons l'analyse, nous nous rendons compte que c'est en même temps ce défaut de rétentionalité qui le rend perpétuellement disponible pour des manipulations futures. Ainsi, l'impossibilité de fixer quoique ce soit est aussi ce qui permet l'exercice indéfini de l'imagination.

Cette caractéristique peut-elle être retrouvée dans l'ensemble des dispositifs ludiques, qu'ils soient formels ou informels? En tout cas, on peut noter qu'il s'agit d'une caractéristique partagée avec le numérique. Nous l'avons déjà souligné : le noème du numérique est le "ça a été manipulé ». Tant et si bien que tout ce qui peut y être construit repose sur des fondations susceptibles à tout moment d'être remises en jeu. Si l'on en vient maintenant au jeu vidéo, on voit que nombre de propositions existantes sont bâties sur cette "faille" du numérique. C'est en particulier le cas des jeux impliquant de conserver la trace de ce qui a été accompli, ce qu'on appelle communément la sauvegarde. On peut s'en convaincre en s'intéressant tout d'abord aux jeux d'arcade, où les seules traces que le joueur peut laisser sont ses initiales, et cela plus encore sous deux conditions: avoir fait un score supérieur aux scores précédents; ne pas voir son score dépassé par un joueur futur. On pourrait bien sûr souligner qu'il s'agit là d'un principe lié à une limitation technique: les capacités limitées des premières machines. Pourtant, ce principe n'a pas disparu. Parmi les nombreux exemples existant, l'un des plus remarquables est le jeu Resident Evil (Capcom, 1996). Dans ce jeu d'aventure horrifique, le joueur ne peut sauvegarder qu'à condition de remplir deux conditions bien précises: se trouver devant l'une des machines à écrire cachées dans le jeu; disposer dans son inventaire d'un ruban encreur. Mais l'action de sauvegarde n'est pas seulement conditionnée dans Resident Evil. Elle implique la destruction du ruban utilisé pour la sauvegarde. Cette ressource étant rare, un dilemme bien connu des joueurs s'installe : faut-il utiliser l'un des rares rubans que l'on possède - au risque de se retrouver sans possibilité de sauvegarder plus tard dans une zone du jeu plus difficile - ou faut-il prendre le risque de continuer à avancer sans sauvegarder, au risque de perdre toute sa progression? Voilà donc la dernière hypothèse que nous voulions poser : l'idée d'une connexion entre jeu et oubli.

\section{Conclusion}

L'essence de la technique ne réside pas en son utilité. La technique n'est pas tant une chose disponible qu'un principe configurateur. Lorsque ce dernier se stabilise, il est aisé de nous y habituer. C'est ce qu'on appelle une époque. Il est alors d'autant plus facile de naturaliser et d'essentialiser nos facultés. A certains égards, la technologie moderne nous sort de ce sommeil dogmatique. Le bouleversement civilisationnel amené par l'arrivée du numérique nous y contraint. Soudain, les choses cessent de nous apparaître familières. Nous ne les comprenons plus. Nous ne nous comprenons plus. Un seul domaine semble épargné par ce moment de déstabilisation: le jeu. Loin d'être affecté par la propagation du numérique, le jeu s'en est emparé avec une facilité déconcertante. C'est que le jeu n'est rien d'autre que déstabilisation, remise en jeu de l'ordre établi. Telle est la raison des difficultés rencontrées par la théorie, quand elle cherche à le définir et le catégoriser. Par nature, le jeu ne peut pas coller avec une conception utilitariste de la technique : cela impliquerait de rencontrer celle-ci comme un support orthothétique, apte à se faire oublier. Or ce que cherche le joueur dans la 
technique est justement l'inverse: non pas des cardinalités et des calendarités permettant de s'orienter, mais la désorientation, l'expérience du labyrinthe. Pour les mêmes raisons, le jeu ne peut se laisser assimiler à une expérience de l'imagination du moins pas tant que celle-ci est comprise comme une forme de mise à distance, de désengagement, c'est-à-dire comme une forme d'expérience spectatoriale.

\section{BIBLIOGRAPHIE}

ADORNO T. \& HORKHEIMER M. (1947), La Dialectique de la Raison, Paris, Gallimard.

ARISTOTE (1994), Éthique à Nicomaque, Paris, Vrin.

BACHIMONT B. (2001), «L'archive numérique : entre authenticité et interprétabilité », Archives, 32-1, pp. 3-15.

BACHIMONT B. (2010), Le sens de la technique : le numérique et le calcul, Paris, Encre Marine.

BJÖRK S. \& JUUL J. (2012), « Zero-Player Games or: What We Talk about When We Talk about Players ", The Philosophy of Computer Games Conference, Madrid, http://www.jesperjuul.net/text/ zeroplayergames/.

CAILLOIS R. (1967), Les jeux et les hommes : le masque et le vertige, Paris, Gallimard.

CHÂTEAU J. (1950), L’Enfant et le jeu, Paris, Éditions du scarabée.

DESCARTES R. (1966), Discours de la méthode, Paris, Gallimard.

ELLUL J. (2012), Le système technicien, Paris, Le Cherche-midi.

FINK E. (1967), Le jeu comme symbole du monde, Paris, Éditions de minuit.

FRASCA G. (2007), Play the message, thèse de doctorat, Institut de Technologie du Danemark.

GOODY J. (1979), La raison graphique. La domestication de la pensée sauvage, Paris, Éditions de minuit.

HENRIOT J. (1969), Le jeu, Paris, Presses Universitaires de France.

HENRIOT J. (1989), Sous couleur de jouer : le métaphorique ludique, Paris, José Corti.

JUUL J. (2011), Half-real, Cambridge, MIT Press.

LEVI-STRAUSS C. (1985), Anthropologie structurale, Paris, Plon.

LEROI-GOURHAN A. (1943), L'homme et la matière, Paris, Albin Michel.

NOVA N. (2013), Joypads ! : Le design des manettes, Lyon, Moutons Électriques.

PLATON (1964), Le Banquet- Phèdre, Paris, Flammarion.

PLATON (1966), La république, Paris, Flammarion.

PLATON (2006), Les Lois, Paris, Flammarion.

PLATON (2018), Gorgias, Paris, Flammarion.

SALEN K. \& ZIMMERMAN E. (2004), Rules of Play: Game Design Fundamentals, Cambridge, MIT Press. 
SIMONDON G. (2012), Du Mode d'existence des objets techniques, Paris, Aubier.

SUITS B. (2005), Grasshopper: Games, Life, and Utopia, Boston, Broadview.

STIEGLER B. (1994), La technique et le temps, tome 1 : La faute d'Épiméthée, Paris, Galilée.

STIEGLER B. (1996), La Technique et le temps, tome 2 : La désorientation, Paris, Galilée.

STIEGLER B. (2001), La technique et le temps, tome 3 : Le temps du cinéma et la question du mal-être, Paris, Galilée.

TRICLOT M. (2011), Philosophie des jeux vidéo, Paris, Zones.

VERNANT J.-P. (2013), Les origines de la pensée grecque, Paris, Presses Universitaires de France.

VIAL S. (2013), L'être et l'écran, Paris, Presses Universitaires de France.

VON NEUMANN J. (1996), L'ordinateur et le cerveau, Paris, Flammarion.

WIENER N. (2014), La cybernétique, Paris, Seuil.

\section{RÉSUMÉS}

Les approches par le game et le play se présentent comme deux voies antithétiques pour les savoirs du jeu. Faut-il comprendre le jeu comme un objet matériel, un système de règles, un dispositif technologique? Ou faut-il plutôt le comprendre l comme une expérience, une forme de vécu ou encore une attitude? A vrai dire, ce dilemme peut se comprendre comme la conséquence d'un présupposé partagé par ces deux approches : soit l'idée d'une opposition entre l'homme et la technique. Dans cet article, il s'agit de montrer en quoi la mobilisation d'une autre conception du rapport de l'homme à la technique, à savoir la thèse de la technique anthropologiquement constitutive et constituante, ouvre des perspectives fécondes pour la compréhension du jeu et du jeu vidéo en particulier.

Game and play are two antithetical approaches. The first one is focused on material, rules or technological devices. The second one relates to experience or attitude. But what if this approach were pre-shapped by a certain idea of technic and human? In this paper, we will suggest that the old division between game and play matches with another division, the traditional opposition between technic and human. Then, we will challenge the possibility that a refine of the relationship between technic and human opens new perspectives about the understanding of the nature of game and play and the meaning of their division.

\section{INDEX}

Mots-clés : jeu, jeu vidéo, homme, technique, philosophie, outil, game, play

Keywords : game, play, human, technic, technology, philosophy, tools, videogame

\section{AUTEUR}

\section{BRICE ROY}

Université de Technologie de Compiègne 